\title{
Functional condition of the hemostasis in newborn calves with signs of iron deficiency, background to ferroglucin
}

\author{
Svetlana $Y u$. Zavalishina* \\ Russian State Social University, Moscow 129226, Russia
}

\begin{abstract}
Further improvement of approaches to breeding and raising cattle is possible with reliance on continuously collected information about its physiology at any age. Their active use in practice is already yielding positive results on the intensification of cattle breeding in different countries of the world. Unfortunately, still not all farms manage to provide optimum housing and feeding for pregnant cows. For this reason, it is still not uncommon for newborn calves to show signs of iron deficiency. In this work, the use of ferroglucin in 38 newborn calves with iron deficiency achieved an improvement in their general condition, normalization of serum iron levels, a slight increase in plasma antioxidant protection levels and a decrease in the intensity of lipid peroxidation in it. Excessive hemocoagulation and platelet activity and reduced hemostatic properties of the vascular wall in the outcome of these calves experienced some positive dynamics against the background of the use of ferroglucin. The weakness of the achieved dynamics of the indicators taken into account indicated the preservation of hemostatic disorders in animals that received ferroglucin, and their risk of microthrombosis, which can slow down their growth and development.
\end{abstract}

\section{Introduction}

Modern physiological science has accumulated a large amount of knowledge about the work of systems and organs of various types of productive animals [1]. Their active use in practice already gives positive results on their breeding, cultivation and treatment $[2,3]$. At the same time, it is not always possible for productive animals to provide optimum feeding and housing conditions [4]. For this reason, various dysfunctions are still often recorded, including in cattle, especially in its early ontogenesis [5]. Unfortunately, one of these disorders is iron deficiency. His attack always negatively affects all life processes in the body of calves, significantly weakening their viability. It is known that iron deficiency [6] has a negative effect on their metabolism, which ultimately inhibits their growth and development. The danger of this condition is also associated with the possibility of hemostasis, leading to the emergence of thrombophilia, on its background [7].

The high prevalence of iron deficiency in newborn calves leads to the frequent occurrence of hemostasis dysfunctions. However, so far in calves, the issues of hemostasis correction in conditions of iron deficiency have not been studied enough. For this reason, the clarification of the possibilities of correcting hemostasiopathy in newborn calves with iron deficiency is of great scientific and practical importance. The elucidation of the influence of ferroglucin traditionally used in the presence of iron deficiency on the state of hemostasis system parameters is of particular interest. In this regard, the study set a goal - to find out the dynamics of hemostasis indicators in newborn calves with iron deficiency who received ferroglucin.

\section{Materials and methods}

The research was conducted in strict accordance with ethical principles established by the European Convent on protection of the vertebrata used for experimental and other scientific purposes (adopted in Strasbourg in March 18, 1986, and confirmed in Strasbourg in June 15, 2006).

The study was performed on 38 newborn calves that had iron deficiency at birth. All calves had erythropoiesis disorders and signs of low iron content in their bodies (the level of serum iron was $12.2 \pm 0.24$ $\mu \mathrm{mol} / \mathrm{l}$, the amount of siderocytes was $1.8 \pm 0.19 \%$, the hemoglobin concentration was $94.9 \pm 0.31 \mathrm{~g} / \mathrm{l}$, the number of erythrocytes $\left.4.4 \pm 0.16 \times 10^{12} / 1\right)$. The control group consisted of 29 healthy newborn calves.

In all animals, plasma lipid peroxidation (LPO) was evaluated by the number of thiobarbituric acid-active products in the course of using the kit produced by AgatMed (Russia), and acylhydroperoxides taking into account the level of plasma antioxidant potential [8]. The concentration of platelets in the blood of animals was determined using a Goryaev chamber. The state of platelet aggregation (AP) was detected by a visual micro-method with a number of inductors: thrombin $(0.125$ units $/ \mathrm{ml})$, ADP $\left(0.5 \times 10^{-4} \mathrm{M}\right)$, adrenaline $\left(5 \times 10^{-6} \mathrm{M}\right)$, collagen $(1: 2$ dilution of the main suspension), ristomycin $(0.8 \mathrm{mg} / \mathrm{ml})$, in terms of standardized by the number of platelets to $200 \times 10^{9}$ platelets in one of its liter.

* Corresponding author: svetlanazsyu@ mail.ru 
The level of antia-ggregation activity of the vessel walls was assessed using a sample with a temporary venous occlusion based on a visual micro-method of AP registration with all inductors used. The value of the anti-aggregation index of the vascular wall was calculated by dividing the time of AP in the plasma after temporary venous stasis by the time of occurrence of AP without it. The value of the index of anticoagulant activity of the vessel wall in the observed calves was determined by dividing the value of the activity of antithrombin III [9] in the plasma obtained after a temporary venous occlusion by its plasma level without it.

The state of vascular control over the blood fibrinolytic activity was determined by calculating the value of the fibrinolytic activity index of the vascular wall during the division of euglobulin lysis time (Barkagan and Momot, 2008) in the plasma obtained without temporal occlusion of the vessels for the lysis time in plasma obtained on its background.

In calves, the duration of activated partial thromboplastin time, prothrombin time, and thrombin time values were recorded [9].

For the correction of iron deficiency in newborn calves, ferroglucin was administered intramuscularly, once, at the rate of $15 \mathrm{mg}$ of iron per $1 \mathrm{~kg}$ of animal body weight. The assessment of the status of the parameters taken into account in healthy animals was carried out once at the age of 6 days. Those who had iron deficiency twice on the first day of life - before using ferroglucin and on the 6th day of life. Statistical processing of the data was carried out by t-student criterion.

\section{Results}

In the outcome of the observed newborn calves that had iron deficiency, weakness, lethargy, lack of interest in the surroundings and pallor of visible mucous membranes were observed.

In calves with iron deficiency, excessive plasma LPO activity was detected (acylhydroperoxide 3.44 \pm 0.25 $\mathrm{D}_{233} / 1 \mathrm{ml}$, thiobarbituric acid-active products $5.20 \pm 0.19$ $\mu \mathrm{mol} / \mathrm{l})$ with a reduced level of antioxidant activity $22.1 \pm 0.27 \%$. The values of the analogous parameters in the control were $1.44 \pm 0.09 \mathrm{D}_{233} / 1 \mathrm{ml}, 3.46 \pm 0.14 \mu \mathrm{mol} / 1$ and $33.7 \pm 0.14 \%$, respectively.

The number of platelets in the blood of newborn calves with iron deficiency corresponded to the norm. At the same time, their AP developed rapidly (table). First of all, AP came in response to collagen $(19.8 \pm 0.15 \mathrm{~s})$, somewhat later on ADP and ristomycin, and still later in response to thrombin $(37.5 \pm 0.07 \mathrm{~s})$. The most delayed in calves with iron deficiency in AP occurred under the influence of adrenaline.

Table 1. Dynamics of hemostasis activity in newborn calves with iron deficiency treated with ferroglucin

\begin{tabular}{|c|c|c|c|}
\hline \multirow[t]{2}{*}{ Registered indicators } & \multicolumn{2}{|c|}{ Calves that received ferroglucin correction, $\mathrm{n}=38, \mathrm{M} \pm \mathrm{m}$} & \multirow{2}{*}{$\begin{array}{c}\text { Control group, } \\
\mathrm{n}=29, \mathrm{M} \pm \mathrm{m}\end{array}$} \\
\hline & exodus & after correction & \\
\hline Platelet aggregation with ADP, $\mathrm{s}$ & $25.6 \pm 0.11$ & $\begin{array}{c}28.6 \pm 0.05 \\
\mathrm{p}_{1}<0.05\end{array}$ & $\begin{array}{c}40.2 \pm 0.08 \\
\mathrm{p}<0.01\end{array}$ \\
\hline Platelet aggregation with collagen, $\mathrm{s}$ & $19.8 \pm 0.15$ & $\begin{array}{c}21.8 \pm 0.07 \\
\mathrm{p}_{1}<0.05\end{array}$ & $\begin{array}{c}31.4 \pm 0.08 \\
\mathrm{p}<0.01\end{array}$ \\
\hline Platelet aggregation with thrombin, $\mathrm{s}$ & $37.5 \pm 0.07$ & $39.1 \pm 0.12$ & $\begin{array}{c}53.8 \pm 0.07 \\
\mathrm{p}<0.01\end{array}$ \\
\hline Platelet aggregation with ristomycin, $\mathrm{s}$ & $23.0 \pm 0.12$ & $\begin{array}{l}25.3 \pm 0.10 \\
\mathrm{p}_{1}<0.05\end{array}$ & $\begin{array}{c}48.0 \pm 0.12 \\
\mathrm{p}<0.01\end{array}$ \\
\hline Platelet aggregation with adrenaline, $\mathrm{s}$ & $70.6 \pm 0.14$ & $\begin{array}{l}78.8 \pm 0.14 \\
\mathrm{p}_{1}<0.05\end{array}$ & $\begin{array}{l}97.6 \pm 0.06 \\
p<0.01\end{array}$ \\
\hline $\begin{array}{l}\text { The value of the index of anticoagulant activity of the } \\
\text { vascular wall with ADP }\end{array}$ & $1.45 \pm 0.12$ & $1.45 \pm 0.07$ & $\begin{array}{c}1.67 \pm 0.12 \\
\mathrm{p}<0.01\end{array}$ \\
\hline $\begin{array}{l}\text { The value of the index of anticoagulant activity of the } \\
\text { vascular wall with collagen }\end{array}$ & $1.36 \pm 0.05$ & $1.35 \pm 0.05$ & $\begin{array}{c}1.58 \pm 0.02 \\
\mathrm{p}<0.01\end{array}$ \\
\hline $\begin{array}{l}\text { The value of the index of anticoagulant activity of the } \\
\text { vascular wall with thrombin }\end{array}$ & $1.40 \pm 0.08$ & $1.45 \pm 0.05$ & $\begin{array}{l}1.52 \pm 0.05 \\
\mathrm{p}<0.01\end{array}$ \\
\hline $\begin{array}{l}\text { The value of the index of anticoagulant activity of the } \\
\text { vascular wall with ristomycin }\end{array}$ & $1.45 \pm 0.06$ & $1.45 \pm 0.05$ & $\begin{array}{c}1.51 \pm 0.04 \\
\mathrm{p}<0.05\end{array}$ \\
\hline $\begin{array}{l}\text { The value of the index of anticoagulant activity of the } \\
\text { vascular wall with adrenaline }\end{array}$ & $1.42 \pm 0.08$ & $1.44 \pm 0.03$ & $\begin{array}{c}1.64 \pm 0.07 \\
\mathrm{p}<0.01\end{array}$ \\
\hline $\begin{array}{l}\text { The value of the index of anticoagulant activity of the } \\
\text { vascular wall }\end{array}$ & $1.23 \pm 0.02$ & $1.24 \pm 0.03$ & $\begin{array}{c}1.31 \pm 0.03 \\
\mathrm{p}<0.01\end{array}$ \\
\hline $\begin{array}{l}\text { The index value of the fibrinolytic activity of the } \\
\text { vascular wall }\end{array}$ & $1.20 \pm 0.08$ & $1.22 \pm 0.03$ & $\begin{array}{c}1.39 \pm 0.10 \\
\mathrm{p}<0.01\end{array}$ \\
\hline The indicator of activated partial thromboplastin time, $\mathrm{s}$ & $27.8 \pm 0.25$ & $29.1 \pm 0.34$ & $\begin{array}{l}39.7 \pm 0.34 \\
\mathrm{p}<0.01\end{array}$ \\
\hline Prothrombin time, $\mathrm{s}$ & $12.6 \pm 0.18$ & $12.9 \pm 0.24$ & $\begin{array}{c}17.4 \pm 0.23 \\
\mathrm{p}<0.01\end{array}$ \\
\hline Thrombin time indicator, $\mathrm{s}$ & $16.6 \pm 0.28$ & $16.7 \pm 0.16$ & $\begin{array}{c}17.2 \pm 0.21 \\
\mathrm{p}<0.01\end{array}$ \\
\hline
\end{tabular}

Note: $p$ - reliability of differences in indicators between the control and the initial state of calves, $\mathrm{p}_{1}$ - reliability of the dynamics of the indicators taken into account in the background of the correction.

In the observed calves with iron deficiency, a decrease in the index of anti-aggregatory activity of the vascular wall was recorded in relation to all inductors used (table 1). The lowest was the value of this index 
with collagen. The index with adrenaline and thrombin was slightly higher; the values of the anti-aggregation activity index of the vascular wall with ADP and ristomycin were even higher.

In the observed newborn calves with iron deficiency, a decrease in the anticoagulant capacity of the vascular wall was observed, as indicated by a decrease in the value of the index of anticoagulant activity of the vascular wall. The fibrinolytic activity of the vessels in these animals was also weakened (the index of the fibrinolytic activity of the vascular wall in them was reduced by $13.7 \%$ ).

For calves with iron deficiency, it was characteristic of accelerating the clotting time along the outer path (by $29.9 \%$ ) and the inner path (by $27.5 \%$ ), as well as some intensification of fibrin formation.

The use of ferroglucin in calves with iron deficiency provided an improvement in their general state indicators, an increase in the amount of serum iron to the normal level, and a slight positive trend in the hemostatic parameters taken into account.

The observed calves showed a slight decrease in the content of plasma acylhydroperoxide $\left(2.45 \pm 0.21 \mathrm{D}_{233} / 1\right.$ $\mathrm{ml})$ and thiobarbituric acid-active products $(4.39 \pm 0.16$ $\mu \mathrm{mol} / \mathrm{l})$ with a slight increase in its level antioxidant activity $(26.9 \pm 0.08 \%)$.

The use of ferroglucin was accompanied in calves with iron deficiency by a slight inhibition of AP. At the same time, the platelets of these animals reacted most actively to collagen, ristomycin and ADP, less actively to thrombin and adrenaline (table).

In the observed calves that received ferroglucin, the absence of significant changes in the values of the index of anti-aggregatory activity of the vascular wall with all the inductors used was noted (table). They had the lowest index with collagen; the values of other indices of anti-aggregatory activity of the vascular wall were slightly higher.

In newborn calves with iron deficiency who received an iron preparation, there was a tendency to increase the value of the index of anticoagulant activity of the vascular wall and the value of the index of fibrinolytic activity of the vascular wall.

As a result of the conducted exposure, weak inhibition of the activated partial thromboplastin time (by $4.5 \%$ ) and prothrombin time (by $2.3 \%$ ) was found. At the same time, the value of thrombin time, which determines the intensity of the transition of fibrinogen to fibrin, in calves treated with ferroglucin was left unchanged.

\section{Discussion}

The overall viability of the organism depends on the mass of environmental [10] and hereditary factors [11]. Their summation in each particular case creates a special situation with the reactivity of the organism [12], its biological characteristics [13] and productive qualities [14]. It has long been recognized that blood plays a huge role in the implementation of protective, adaptive and pathological processes [15]. It is a fluid medium of the body, providing its self-regulation [16]. It is very important to maintain the hemostasis of the body is the hemostatic system that regulates many processes in the blood [17, 18].

In previous studies, it was noted that the optimal course of ontogenesis is always associated with a low activity of the components of the hemostasis system [19]. Any violation of hemostasis and the occurrence of various deficient situations for any substances, as a rule, are accompanied by dysfunction of many organs and systems [20], including the hemostasis system [21].

In the study, it was found that depression of the antioxidant protection of the plasma of newborn calves with iron deficiency contributes to the activation of lipid peroxidation in plasma. This process damages the structures of blood plates and blood vessels [22] and severely disrupts their functions [23]. An increase in newborn calves with iron-deficient antibodies found in the work indicates an increase in platelet sensitivity to external stimulating effects [24]. The increase in AP in response to ristomycin in calves with iron deficiency indicates an increase in their sensitivity to von Willebrand factor [25]. Acceleration of AP with ADP in these animals indicates an increase in the intensity of arachidonic acid conversion into a blood platelet into a powerful platelet aggregation stimulator, thromboxane [26].

The weakening of vascular hemostasis in calves with iron deficiency was revealed in them by lowering the antiaggregatory capacity of the vessels. Apparently, this is based on a reduction in the production of prostacyclin and nitric oxide in their walls. In addition, a prominent role in the occurrence of vasopathy in these animals belongs to the weakening of the anticoagulant and fibrinolytic properties of the vascular endothelium as a result of weakening the production of antithrombin III and tissue plasminogen activators in it [27].

The found acceleration of prothrombin time in the examined animals with iron deficiency reflected in them the intensification of the mechanisms of plasma hemostasis activation along the external coagulation pathway. It was largely due to the increased formation in the blood of these calves highly active thromboplastin [28]. The rapidity of hemocoagulation in the activated partial thromboplastin time assay indicated an increase in the functioning of the internal coagulation pathway. The acceleration of the final hemocoagulation stage in the observed calves was indicated by shortening of the thrombin time [29].

The use of ferroglucin caused in newborn calves that had dysfunction at birth, iron dysfunction, their body saturation with iron. This improved the performance of their red blood and the general condition of the animals.

The use of ferroglucin contributed to a decrease in the liquid part of blood in calves with iron deficiency in the intensity of the lipid peroxidation process. This weakened its stimulating effect on the surface structures of platelets. Non-expressed inhibition of AP in calves that had an iron deficiency as a result of the use of ferroglucin is largely a consequence of the weakness of positive changes on the background of the correction for lipid peroxidation, as well as on the receptor and 
postreceptor mechanisms of platelet function. Nonexpressed prolongation of the time of occurrence of AP under the action of ristomycin indicated the possibility of a slight decrease in the blood of calves treated with ferroglucin, the level of adhesion cofactor von Willebrand factor [30].

As a result of the correction in animals with iron deficiency, some enhancement of the antiaggregation, anticoagulant and fibrinolytic properties of the vascular wall was noted. This was associated with a slight intensification of the production of prostacyclin, nitric oxide, antithrombin III and tissue plasminogen activators in these animals in its endothelium [8].

The slowing down of prothrombin time found in calftreated calves reflected the positive dynamics of the mechanisms of plasma hemostasis activation along the external pathway, which was developing and was largely due to the weakening of their activity that triggers the clotting of thromboplastin [5]. A certain inhibition of the initially accelerated activated partial thromboplastin time revealed on the background of the correction indicated a decrease in the activity of the internal coagulation pathway. The inhibition of the final stage of hemocoagulation in these animals was indicated by an increase in their thrombin time.

Thus, in newborn calves with an iron deficiency state, hemostatic abilities of platelets and plasma hemostasis are observed, while control over them from the vascular wall is weakened. The use of ferroglucin in these animals can cause only a small positive dynamics of the recorded parameters of hemostasis.

\section{Conclusion}

In newborn calves with iron deficiency, a decrease in the activity of plasma antioxidant protection and an increase in the intensity of lipid peroxidation in it are characteristic. This is accompanied by their increased hemostatic properties of platelets and blood clotting activity and weakening the ability of the vascular wall to restrain them. In the case of ferroglucin in newborn calves with signs of iron deficiency, there is some increase in plasma antioxidant protection, weakening of lipid peroxidation, a slight decrease in the activity of platelet and plasma components of hemostasis and a slight increase in hemostatic properties of the vascular wall. The persistence of hemostatic disorders in animals treated with ferroglucin and iron deficiency indicated that they had a risk of microthrombosis capable of inhibiting the processes of growth and development.

\section{References}

1. L. Burnier, P. Fontana, B.R. Kwak, A. AngelilloScherrer, Cell-derived microparticles in haemostasis and vascular medicine, J. Thromb. Haemost, 101, 439-451 (2009)

2. L.V. Korepanova, O.S. Starostina, S.D. Batanov, The Blood as an indicator of the interior features of the hybrid animals, Husbandry, 10, 26-28 (2015)
3. A.A. Bikbulatova, Creating Psychological Comfort In Women Who Wear Corrective Clothing For A Long Time, Res. J. of Pharmaceut., Biolog. and Chemical Sci., 9(6), 1112-1121 (2018)

4. H. Kulig, K. Żukowski, I. Kowalewska-Łuczak, P. Łakomy, ScdlPolymorphism and Breeding Value for Milk Production Traits in Cows, Bulgar. J. of Agricult. Sci., 22, 131-134 (2016)

5. I.N. Medvedev, Functional Features Of Platelet Hemostasis In Athletes-Athletes 18-35 Years, Res. J. of Pharmaceut., Biolog. and Chemical Sci., 9(6), 1196-1201 (2018)

6. N.V. Vorobyeva, I.N. Medvedev, Functional activity of platelets in new-born calves of blackmarked breed, Bulgar. J. of Agricult. Sci., 25(3), 570-574 (2019)

7. G.N. Sushkevich, Abnormal hemostasis system and the principles of their correction (Sovetskaya Kuban, Krasnodar, 2010)

8. I.A. Volchegorsky, I.I. Dolgushin, O.L. Kolesnikov, V.E. Tseylikman, Experimental modeling and laboratory evaluation of adaptive reactions of the body (Chelyabinsk, 2000)

9. Z.S. Barkagan, A.P. Momot, Diagnosis and controlled therapy of hemostatic disorders (Newdiamed, Moscow, 2008)

10. I.N. Medvedev, Physiological Activity Of The Blood Plates In Regularly Practicing Amateur Football Players, Res. J. of Pharmaceut., Biolog. and Chemical Sci., 9(6), 1161-1166 (2018)

11. I.N. Medvedev, Dynamics Of Functional Parameters Of Platelet Hemostasis In Young People With Hemodynamic And Metabolic Disorders On The Background Of Regular Physical Activity, Res. J. of Pharmaceut., Biolog. and Chemical Sci., 9(6), 1217-1222 (2018)

12. G.C. White, R. Rompietti, Platelet secretion: indiscriminately spewed forth or highly orchestrated? J. Thromb. Haemost, 5, 2009-2016 (2007)

13. Ju.L. Oshurkova, I.N. Medvedev, Physiological Indicators Of Platelets In Ayrshire Calves During The Dairy Feeding Phase, Res. J. of Pharmaceut., Biolog. and Chemical Sci., 9(6), 171-176 (2018)

14. I.N. Medvedev, Physical Effect Of Feasible Physical Exertion On Platelet Activity In Overweight Young Men, Res. J. of Pharmaceut., Biolog. and Chemical Sci., 9(6), 1137-1142 (2018)

15. I.N. Medvedev, The Physiological Properties Of Platelets In People 18-35 Years Old, Trained In The Section Of General Physical Training, Res. J. of Pharmaceut., Biolog. and Chemical Sci., 9(6), 1277-1283 (2018)

16. I.N. Medvedev, Platelet Functions In The First Adulthood When Regularly Practiced In Adolescence In The Tennis Section, Res. J. of Pharmaceut., Biolog. and Chemical Sci., 9(6), 1180-1184 (2018) 
17. N.V. Vorobyeva, I.N. Medvedev, Physiological Features Of Platelet Functioning In Calves of Holstein Breed During The Newborn, Res. J. of Pharmaceut., Biolog. and Chemical Sci., 9(6), 129-135 (2018)

18. I.N. Medvedev, Physiological Response of Intravascular Platelet Activity In Boys With High Normal Blood Pressure To Regular Physical Exercise, Res. J. of Pharmaceut., Biolog. and Chemical Sci., 9(6), 1244-1250 (2018)

19. E.M. Lenchenko, Yu.A. Vatnikov, E.V. Kulikov, D.A. Lozovoy, V.A. Gavrilov, L.A. Gnezdilova, V.N. Zimina, V.I. Kuznetsov, V.V. Annikov, I.N. Medvedev, A.V. Petryaeva, T.I. Glagoleva, Aspects of Salmonellosis pathogenesis using chicken models, Bali Med. J., 8(1), 206-210 (2019) DOI: 10.15562/bmj.v8i1.920

20. I.N. Medvedev, Functional Features Of Intravascular Platelet Activity In Adolescents With High Normal Blood Pressure, Overweight Or A Combination Of Them Against The Background Of Regular Physical Exertion, Res. J. of Pharmaceut., Biolog. and Chemical Sci., 9(6), 1258-1265 (2018)

21. A.A. Bikbulatova, Functional Features of Microcirculatory Processes In Obese Women Against A Background Of Long Daily Wearing Of Corrective Clothing, Res. J. of Pharmaceut., Biolog. and Chemical Sci., 9(6), 785-793 (2018)

22. I.N. Medvedev, Vascular-platelet interaction in pregnant cows, Bulg. J. Agric. Sci, 23(2), 310-314 (2017)

23. Ju.L. Oshurkova, I.N. Medvedev, Functional Features Of Platelets In Newborn Calves Ayrshire Breed, Res. J. of Pharmaceut., Biolog. and Chemical Sci., 9(6), 313-318 (2018)
24. I.N. Medvedev, Physiological Characteristics Of Platelet Activity In Young People Experiencing Moderate Exercise, Res. J. of Pharmaceut., Biolog. and Chemical Sci., 9(6), 1416-1421 (2018)

25. I.N. Medvedev, The Physiological State Of Intravascular Platelet Activity In Young Men Who Had High Normal Blood Pressure, Overweight Or A Combination Of Them And Started Regular Exercise, Res. J. of Pharmaceut., Biolog. and Chemical Sci., 9(6), 1438-1445 (2018)

26. I.N. Medvedev, Physiological Effects Of Physical Stress On Platelet Hemostasis In Young Individuals With High Normal Blood Pressure And Overweight, Res. J. of Pharmaceut., Biolog. and Chemical Sci., 9(6), 1466-1471 (2018)

27. I.N. Medvedev, Physiological Response Of Platelet Activity In Young People With High Normal Blood Pressure To Regular Exercise, Res. J. of Pharmaceut., Biolog. and Chemical Sci., 9(6), 1489-1494 (2018)

28. I.N. Medvedev, O.A. Danilenko, Complex correction of vascular hemostasis in patients with arterial hypertension, metabolic syndrome, and recent ocular vessel occlusion, Russ. J. of Cardiol., 4, 15-19 (2010)

29. A.S. Shitikova, Trombotsitopatii of congenital and acquired (St. Petersburg, 2008)

30. I.A. Skoryatina, I.N. Medvedev, Correction of aggregation level of basic regular blood elements in patients with hypertension and dyslipidemia receiving rosuvastatin and non-medicinal treatment, Bali Med. J., 8(1), 194-200 (2019) DOI: 10.15562/bmj.v8i1.648 